\title{
On Decompositions of Continuity and $\alpha$-Continuity
}

\author{
ZBIGNIEW DUSZYŃSKI
}

Abstract. Several results concerning a decomposition of $\alpha$-continuous, continuous and complete continuous functions are offered.

\section{Preliminaries}

Throughout the paper, by $(X, \tau)$ and $(Y, \sigma)$ we denote topological spaces (briefly: spaces) on which no separation axioms are assumed. For a subset $S$ of a space $(X, \tau), \operatorname{cl}(S)$ and $\operatorname{int}(S)$ denote the closure and the interior of $S$, respectively. A set $S \subset X$ is called regular open (resp. regular closed) if $S=\operatorname{int}(\operatorname{cl}(S))(\operatorname{resp} . S=\operatorname{cl}(\operatorname{int}(S)))$. An $S \subset X$ is said to be $\alpha$-open [9] (resp. preopen [8], semi-open [7], semi-preopen [1]) if $S \subset \operatorname{int}(\operatorname{cl}(\operatorname{int}(S)))$ (resp. $S \subset \operatorname{int}(\operatorname{cl}(S)), S \subset \operatorname{cl}(\operatorname{int}(S)), S \subset \operatorname{cl}(\operatorname{int}(\operatorname{cl}(S))))$.

The family $\tau^{\alpha}$ of all $\alpha$-open subsets of a space $(X, \tau)$ is always a topology on $X[9]$, such that $\tau^{\alpha} \supset \tau$ (the inclusion is proper, in general). Crossley and Hildebrand [3] investigated semi-closed subsets of $(X, \tau): S$ is semi-closed if $S \supset \operatorname{int}(\operatorname{cl}(S))$. They have obtained that $S$ is semiclosed in $(X, \tau)$ if and only if int $(\operatorname{cl}(S))=\operatorname{int}(S)$. Using this identity, Tong [12] introduced the so-called $t$-sets. He proved that each regular open set is a $t$-set [12, Proposition 2]. The family of all regular open (resp. regular closed, closed, semi-open, semi-closed, preopen, semi-preopen) subsets of $(X, \tau)$ is denoted as $\mathrm{RO}(X, \tau)$ (resp. $\mathrm{RC}(X, \tau), \mathrm{c}(X, \tau), \mathrm{SO}(X, \tau)$, $\operatorname{SC}(X, \tau), \operatorname{PO}(X, \tau), \operatorname{SPO}(X, \tau))$. The following inclusions (proper in general) are known:

- $\mathrm{RO}(X, \tau) \subset \tau \subset \tau^{\alpha} \subset \mathrm{PO}(X, \tau) \subset \operatorname{SPO}(X, \tau)$,

- $\tau^{\alpha} \subset \operatorname{SO}(X, \tau) \subset \operatorname{SPO}(X, \tau)$.

The families $\operatorname{PO}(X, \tau)$ and $\mathrm{SO}(X, \tau)$ are, in general, independent of each other in the sense of inclusion [10].

2010 Mathematics Subject Classification. Primary: 54A05.

Key words and phrases. Preopen set, $\alpha$-open set, semi-open set, $\alpha$-precontinuity, continuity, complete continuity. 
Lemma 1.1 ([10] for "semi-open", [4] for "semi-closed"). If either $S_{1} \in$ $\mathrm{SO}(X, \tau) \cup \mathrm{SC}(X, \tau)$ or $S_{2} \in \mathrm{SO}(X, \tau) \cup \mathrm{SC}(X, \tau)$, then

$$
\operatorname{int}\left(\operatorname{cl}\left(S_{1} \cap S_{2}\right)\right)=\operatorname{int}\left(\operatorname{cl}\left(S_{1}\right)\right) \cap \operatorname{int}\left(\operatorname{cl}\left(S_{2}\right)\right) .
$$

Lemma 1.2 ([10]). In any space $(X, \tau), \tau^{\alpha}=\operatorname{PO}(X, \tau) \cap \operatorname{SO}(X, \tau)$.

\section{Continuity}

We will need the following classes of subsets of a space $(X, \tau)$.

Definition 2.1. $\quad(\tau 0) \mathcal{B}_{\tau}^{0}(X, \tau):=\{S \subset X: S=U \cap C, U \in \tau, C \in$ $\mathrm{RO}(X, \tau)\}$

$(\tau 1) \mathcal{B}_{\tau}^{1}(X, \tau):=\{S \subset X: S=U \cap C, U \in \tau, C \in \operatorname{RC}(X, \tau)\}(=\mathcal{A}(X, \tau)$ [11, Definition 3.1] and [12, p. 31]);

$(\tau 2) \mathcal{B}_{\tau}^{2}(X, \tau):=\{S \subset X: S=U \cap C, U \in \tau, C \in \mathrm{c}(X, \tau)\}(=\mathrm{LC}(X, \tau)$ $[5])$

$(\tau 3) \mathcal{B}_{\tau}^{3}(X, \tau):=\left\{S \subset X: S=U \cap C, U \in \tau, C \in \mathrm{c}\left(X, \tau^{\alpha}\right)\right\}$;

$(\tau 4) \mathcal{B}_{\tau}^{4}(X, \tau):=\{S \subset X: S=U \cap C, U \in \tau, C \in \operatorname{SC}(X, \tau)\}(=\mathcal{B}(X, \tau)$ [12, Definition 2]).

The following (obvious) inclusions, proper in general, hold:

$$
\mathcal{B}_{\tau}^{1}(X, \tau) \subset \mathcal{B}_{\tau}^{2}(X, \tau) \subset \mathcal{B}_{\tau}^{3}(X, \tau) \subset \mathcal{B}_{\tau}^{4}(X, \tau) .
$$

In Theorem 2.1 we recall some results that have been so far obtained.

Theorem 2.1. Let $(X, \tau)$ be a topological space.

(a) $\tau=\operatorname{PO}(X, \tau) \cap \mathcal{A}(X, \tau)$ [6, case (iv), p. 31];

(b) $\tau=\mathrm{PO}(X, \tau) \cap \mathrm{LC}(X, \tau)[6$, Theorem 2(3)];

(c) $\tau=\mathrm{PO}(X, \tau) \cap \mathcal{B}(X, \tau)$ [12, Proposition 9].

We complete these decompositions of $\tau$ in the theorem below.

Theorem 2.2. Let $(X, \tau)$ be a topological space.

(d) $\tau=\operatorname{PO}(X, \tau) \cap \mathcal{B}_{\tau}^{0}(X, \tau)$;

(e) $\tau=\operatorname{PO}(X, \tau) \cap \mathcal{B}_{\tau}^{3}(X, \tau)$.

Proof. Let $S \in \mathrm{PO}(X, \tau) \cap \mathcal{B}_{\tau}^{0}(X, \tau)$. Then $S \in \mathrm{PO}(X, \tau)$ and $S \in \mathcal{B}_{\tau}^{0}(X, \tau) \subset$ $\mathcal{B}(X, \tau)$ since $\operatorname{RO}(X, \tau) \subset \mathrm{SC}(X, \tau)$. By [12, Proposition 9] we have $S \in \tau$. That $S \in \tau$ follows from $S \in \operatorname{PO}(X, \tau) \cap \mathcal{B}_{\tau}^{3}(X, \tau)$ is analogous because $\mathrm{c}\left(X, \tau^{\alpha}\right) \subset \mathrm{SC}(X, \tau)$.

Let now $S \in \tau$. Then $S \in \mathrm{PO}(X, \tau)$ and $S=S \cap X$, where $X \in \mathrm{RO}(X, \tau)$. For (e), observe that $X \in \mathrm{c}\left(X, \tau^{\alpha}\right)$.

Definition 2.2. A function $f:(X, \tau) \rightarrow(Y, \sigma)$ is called $\mathcal{B}_{\tau}^{i}$-continuous on $(X, \tau), i=0,1,2,3,4$, if $f^{-1}(V) \in \mathcal{B}_{\tau}^{i}(X, \tau)$ for any $V \in \sigma$.

For $i=1,2,4$ these types of continuity were introduced earlier: $\mathcal{A}$ continuity ( $\equiv \mathcal{B}_{\tau}^{1}$-continuity) [11], LC-continuity ( $\equiv \mathcal{B}_{\tau}^{2}$-continuity) [6], $\mathcal{B}$ continuity ( $\equiv \mathcal{B}_{\tau}^{4}$-continuity) [12].

By Theorems 2.1 and 2.2 we get the following decomposition results. 
Theorem 2.3. Let $f:(X, \tau) \rightarrow(Y, \sigma)$ be a function. Then, for each $i=$ $0,1,2,3,4$,

$\left(\mathrm{a}_{i}\right) f$ is continuous if and only if $f$ is precontinuous and $\mathcal{B}_{\tau}^{i}$-continuous.

Decompositions of continuity for cases $\left(\mathrm{a}_{1}\right),\left(\mathrm{a}_{2}\right)$ and $\left(\mathrm{a}_{4}\right)$ were known before: [6, Theorem 4(v)], [6, Theorem 4(iv)] and [12, Proposition 11], respectively.

\section{Complete continuity}

Definition 3.1. Let $(X, \tau)$ be a topological space.

$(r \tau 1) \mathcal{B}_{r \tau}^{1}(X, \tau):=\{S \subset X: S=U \cap C, U \in \operatorname{RO}(X, \tau), C \in \operatorname{RC}(X, \tau)\}$;

$(r \tau 2) \mathcal{B}_{r \tau}^{2}(X, \tau):=\{S \subset X: S=U \cap C, U \in \operatorname{RO}(X, \tau), C \in \mathrm{c}(X, \tau)\}$;

$(r \tau 3) \mathcal{B}_{r \tau}^{3}(X, \tau):=\left\{S \subset X: S=U \cap C, U \in \operatorname{RO}(X, \tau), C \in \mathrm{c}\left(X, \tau^{\alpha}\right)\right\}$;

$(r \tau 4) \mathcal{B}_{r \tau}^{4}(X, \tau):=\{S \subset X: S=U \cap C, U \in \operatorname{RO}(X, \tau), C \in \operatorname{SC}(X, \tau)\}$.

Theorem 3.1. For any topological space $(X, \tau)$,

$\left(\mathrm{a}_{r 4}\right)$ a set $S \in \mathrm{RO}(X, \tau)$ if and only if $S \in \mathrm{PO}(X, \tau)$ and $S \in \mathcal{B}_{r \tau}^{4}(X, \tau)$.

Proof. $(\Rightarrow)$ Let $S \in \mathrm{RO}(X, \tau)$. Then $S \in \mathrm{PO}(X, \tau)$ and $S=S \cap X$ where $X \in \mathrm{SC}(X, \tau)$.

$(\Leftarrow)$ If $S \in \operatorname{PO}(X, \tau) \cap \mathcal{B}_{r \tau}^{4}(X, \tau)$, then $S \subset \operatorname{int}(\operatorname{cl}(U \cap C))$ where $U \in$ $\mathrm{RO}(X, \tau)$ and $C \in \mathrm{SC}(X, \tau)$. Using Lemma 1.1 we get

$$
S \subset \operatorname{int}(\operatorname{cl}(U)) \cap \operatorname{int}(\operatorname{cl}(C))=U \cap \operatorname{int}(C),
$$

since $C \in \mathrm{SC}(X, \tau)$ (or by $U \in \operatorname{RO}(X, \tau))$. Then we have:

$$
S=(U \cap C) \cap U \subset(U \cap \operatorname{int}(C)) \cap U=U \cap \operatorname{int}(C) .
$$

Since $S \supset U \cap \operatorname{int}(C)$, we get $S=U \cap \operatorname{int}(C)=U \cap \operatorname{int}(\operatorname{cl}(C))$. But sets $U, \operatorname{int}(\operatorname{cl}(C)) \in \operatorname{RO}(X, \tau)$. Therefore $S$ is an intersection of two regularly open sets - consequently, $S \in \mathrm{RO}(X, \tau)$.

We introduce now four new types of continuity.

Definition 3.2. A function $f:(X, \tau) \rightarrow(Y, \sigma)$ is said to be $\mathcal{B}_{r \tau}^{i}$-continuous on $(X, \tau), i=1,2,3,4$, if $f^{-1}(V) \in \mathcal{B}_{r \tau}^{i}(X, \tau)$ for every $V \in \sigma$.

Definition 3.3. [2] A function $f:(X, \tau) \rightarrow(Y, \sigma)$ is said to be completely continuous if $f^{-1}(V) \in \mathrm{RO}(X, \tau)$ for every $V \in \sigma$.

By Theorem 3.1 we obtain the following decomposition result:

Theorem 3.2. Let $f:(X, \tau) \rightarrow(Y, \sigma)$ be a function. Then:

$\left(\mathrm{b}_{r 4}\right) f$ is completely continuous if and only if it is both precontinuous and $\mathcal{B}_{r \tau}^{4}$-continuous.

We can get more decompositions of complete continuity. Namely, one easily obtains

Theorem 3.3. Let $(X, \tau)$ be an arbitrary space. Then for $i=1,2,3$, 
$\left(\mathrm{a}_{r i}\right) S \in \mathrm{RO}(X, \tau)$ if and only if $S \in \mathrm{PO}(X, \tau) \cap \mathcal{B}_{r \tau}^{i}(X, \tau)$.

Theorem 3.4. Let $f:(X, \tau) \rightarrow(Y, \sigma)$ be a function. Then, for $i=1,2,3$, the following hold:

$\left(\mathrm{b}_{r i}\right) f$ is completely continuous if and only if $f$ is precontinuous and $\mathcal{B}_{r \tau^{-}}^{i}$ continuous.

\section{4. $\alpha$-CONTINUITY}

Definition 4.1. Let $(X, \tau)$ be a topological space.

$(\alpha 0) \mathcal{B}_{\alpha}^{0}(X, \tau):=\left\{S \subset X: S=U \cap C, U \in \tau^{\alpha}, C \in \operatorname{RO}(X, \tau)\right\} ;$

$(\alpha 1) \mathcal{B}_{\alpha}^{1}(X, \tau):=\left\{S \subset X: S=U \cap C, U \in \tau^{\alpha}, C \in \operatorname{RC}(X, \tau)\right\}$;

$(\alpha 2) \mathcal{B}_{\alpha}^{2}(X, \tau):=\left\{S \subset X: S=U \cap C, U \in \tau^{\alpha}, C \in \mathrm{c}(X, \tau)\right\}$;

$(\alpha 3) \mathcal{B}_{\alpha}^{3}(X, \tau):=\left\{S \subset X: S=U \cap C, U \in \tau^{\alpha}, C \in \mathrm{c}\left(X, \tau^{\alpha}\right)\right\}$;

$(\alpha 4) \mathcal{B}_{\alpha}^{4}(X, \tau):=\left\{S \subset X: S=U \cap C, U \in \tau^{\alpha}, C \in \operatorname{SC}(X, \tau)\right\}$.

Theorem 4.1. Let $(X, \tau)$ be arbitrary. Then:

$\left(\mathrm{a}_{\alpha 4}\right) S \in \tau^{\alpha}$ if and only if $S \in \mathrm{PO}(X, \tau) \cap \mathcal{B}_{\alpha}^{4}(X, \tau)$.

Proof. $(\Rightarrow)$ Let $S \in \tau^{\alpha}$. Then $S \in \operatorname{PO}(X, \tau)$ and $S=S \cap C$, where $C=$ $X \in \mathrm{SC}(X, \tau)$.

$(\Leftarrow)$ Suppose $S \in \mathrm{PO}(X, \tau) \cap \mathcal{B}_{\alpha}^{4}(X, \tau)$. Then $S \in \mathrm{PO}(X, \tau)$ and $S=$ $U \cap C$, where $U \in \tau^{\alpha}, C \in \mathrm{SC}(X, \tau)$. Utilizing Lemma 1.1 we obtain

$$
\begin{aligned}
& S \subset \operatorname{int}(\operatorname{cl}(U \cap C))=\operatorname{int}(\operatorname{cl}(U)) \cap \operatorname{int}(\operatorname{cl}(C)) \subset \\
& \quad \subset \operatorname{int}(\operatorname{cl}(\operatorname{int}(\operatorname{cl}(\operatorname{int}(U))))) \cap \operatorname{int}(C)=\operatorname{int}(\operatorname{cl}(\operatorname{int}(U))) \cap \operatorname{int}(C) .
\end{aligned}
$$

Hence

$\left(* *^{\prime}\right) \quad S=(U \cap C) \cap U \subset(\operatorname{int}(\operatorname{cl}(\operatorname{int}(U))) \cap \operatorname{int}(C)) \cap U=U \cap \operatorname{int}(C)$.

On the other hand, $S=U \cap C \supset U \cap \operatorname{int}(C)$. This shows that $S=U \cap$ $\operatorname{int}(C) \in \tau^{\alpha}$.

Observe that $X \in \mathrm{RO}(X, \tau) \cap \mathrm{RC}(X, \tau)$ and $\operatorname{RC}(X, \tau) \subset \mathrm{c}(X, \tau) \subset$ $\mathrm{c}\left(X, \tau^{\alpha}\right) \subset \mathrm{SC}(X, \tau)$. Thus, by the proof of sufficiency of Theorem 4.1, one easily deduces the following result.

Theorem 4.2. Let $(X, \tau)$ be arbitrary. Then for $i=0,1,2,3$ one has:

$\left(\mathrm{a}_{\alpha i}\right) S \in \tau^{\alpha}$ if and only if $S \in \mathrm{PO}(X, \tau) \cap \mathcal{B}_{\alpha}^{i}(X, \tau)$.

For a function $f:(X, \tau) \rightarrow(Y, \sigma)$ we may define the respective notions of $\mathcal{B}_{\alpha}^{i}$-continuity - see Definitions 2.2 or 3.2. Theorems 4.1 and 4.2 lead immediately to

Theorem 4.3. Let $f:(X, \tau) \rightarrow(Y, \sigma)$ be a function. For $i=0,1,2,3,4$, $\left(\mathrm{b}_{\alpha i}\right) f$ is $\alpha$-continuous if and only if $f$ is precontinuous and $\mathcal{B}_{\alpha}^{i}$-continuous.

Definition 4.2. Let $(X, \tau)$ be a space.

$(s 0) \mathcal{B}_{s}^{0}(X, \tau):=\{S \subset X: S=U \cap C, U \in \mathrm{SO}(X, \tau), C \in \operatorname{RO}(X, \tau)\} ;$ 
(s1) $\mathcal{B}_{s}^{1}(X, \tau):=\{S \subset X: S=U \cap C, U \in \mathrm{SO}(X, \tau), C \in \mathrm{RC}(X, \tau)\}$;

$(s 2) \mathcal{B}_{s}^{2}(X, \tau):=\{S \subset X: S=U \cap C, U \in \mathrm{SO}(X, \tau), C \in \mathrm{c}(X, \tau)\}$;

(s3) $\mathcal{B}_{s}^{3}(X, \tau):=\left\{S \subset X: S=U \cap C, U \in \mathrm{SO}(X, \tau), C \in \mathrm{c}\left(X, \tau^{\alpha}\right)\right\}$;

(s4) $\mathcal{B}_{s}^{4}(X, \tau):=\{S \subset X: S=U \cap C, U \in \mathrm{SO}(X, \tau), C \in \mathrm{SC}(X, \tau)\}$.

Theorem 4.4. Let $(X, \tau)$ be a space. Then

$\left(\mathrm{a}_{s 4}\right) S \in \tau^{\alpha}$ if and only if $S \in \mathrm{PO}(X, \tau) \cap \mathcal{B}_{s}^{4}(X, \tau)$.

Proof. Let $S \in \operatorname{PO}(X, \tau) \cap \mathcal{B}_{s}^{4}(X, \tau)$. Hence $S \subset \operatorname{int}(\operatorname{cl}(S))$ and $S=U \cap C$ for $U \in \mathrm{SO}(X, \tau)$ and $C \in \mathrm{SC}(X, \tau)$. We have what follows (using Lemma 1.1):

(*”) $S \subset \operatorname{int}(\operatorname{cl}(U \cap C))=\operatorname{int}(\operatorname{cl}(U)) \cap \operatorname{int}(C) \subset \operatorname{cl}(\operatorname{int}(\operatorname{cl}(U))) \cap \operatorname{int}(C)$.

Since $\operatorname{SO}(X, \tau) \subset \operatorname{SPO}(X, \tau)$,

$(* *) \quad S=(U \cap C) \cap U \subset(\operatorname{cl}(\operatorname{int}(\operatorname{cl}(U))) \cap \operatorname{int}(C)) \cap U=U \cap \operatorname{int}(C)$.

But $S=U \cap C \supset U \cap \operatorname{int}(C)$ and hence $S=U \cap \operatorname{int}(C) \in \operatorname{SO}(X, \tau)$, since the intersection of semi-open and open sets is always semi-open [7].

Thus we have proved that $\mathrm{PO}(X, \tau) \cap \mathcal{B}_{s}^{4}(X, \tau) \subset \mathrm{SO}(X, \tau)$. Since $\tau^{\alpha} \subset$ $\mathrm{PO}(X, \tau) \cap \mathcal{B}_{s}^{4}(X, \tau)$ and, by Lemma 1.2 , we have

$$
\tau^{\alpha} \cap \mathrm{PO}(X, \tau) \subset \mathrm{PO}(X, \tau) \cap \mathcal{B}_{s}^{4}(X, \tau) \subset \mathrm{PO}(X, \tau) \cap \mathrm{SO}(X, \tau)=\tau^{\alpha} .
$$

Therefore $\left(\tau^{\alpha} \subset \mathrm{PO}(X, \tau)\right)$, one obtains $\tau^{\alpha}=\mathrm{PO}(X, \tau) \cap \mathcal{B}_{s}^{4}(X, \tau)$.

Similarly to the observations in the lines preceding Theorem 4.2 one can formulate

Theorem 4.5. Let $(X, \tau)$ be a space. Then for $i=0,1,2,3$,

$\left(\mathrm{a}_{s i}\right) S \in \tau^{\alpha}$ if and only if $S \in \operatorname{PO}(X, \tau) \cap \mathcal{B}_{s}^{i}(X, \tau)$.

For a function $f:(X, \tau) \rightarrow(Y, \sigma)$ one can define the respective notions of $\mathcal{B}_{s}^{i}$-continuity $(i=0,1,2,3,4)$ - we leave it to the reader. The following decomposition results follow from Theorems 4.4 and 4.5.

Theorem 4.6. Let $f:(X, \tau) \rightarrow(Y, \sigma)$, be a function. Then for $i=0,1,2,3,4$, $\left(\mathrm{b}_{s i}\right) f$ is $\alpha$-continuous if and only if $f$ is precontinuous and $\mathcal{B}_{s}^{i}$-continuous.

\section{REFERENCES}

[1] D. Andrijević, Semi-preopen sets, Mat. Vesnik, 38 (1986), 24-32.

[2] S.P. Arya, R. Gupta, On strongly continuous mappings, Kyungpook Math. J., 14 (1974), 131-143.

[3] C.G. Crossley, S. K. Hildebrand, Semi-closure, Texas J. Sci., 22(2-3) (1971), 99-112.

[4] Z. Duszyński, Optimality of mappings and some separation axioms, Rendiconti del Circolo Matematico di Palermo, 57 (2008), 213-228.

[5] M. Ganster, I. L. Reilly, Locally closed sets and LC-continuous functions, Internat. J. Math. Math. Sci., 3 (1989), 417-424.

[6] M. Ganster, I. L. Reilly, A decomposition of continuity, Acta Math. Hungar., 56(3-4) (1990), 299-301. 
[7] N. Levine, Semi-open sets and semi-continuity in topological spaces, Amer. Math. Monthly, 70 (1963), 36-41.

[8] A.S. Mashhour, M. E. Abd El-Monsef, S. N. El-Deeb, On precontinuous and weak precontinuous mappings, Proc. Math. and Phys. Soc. Egypt, 53 (1982), 47-53.

[9] O. Njåstad, On some classes of nearly open sets, Pacific J. Math., 15 (1965), 961-970.

[10] T. Noiri, On $\alpha$-continuous functions, Čas. pěst. mat., 109 (1984), 118-126.

[11] J. Tong, A decomposition of continuity, Acta Math. Hungar., 48(1-2) (1986), 11-15.

[12] J. Tong, On decompositions of continuity in topological spaces, Acta Math. Hungar., 51(1-2) (1989), 51-55.

\section{ZBIGNIEW DUSZYŃSKI}

Casimirus the Great University

Department of Mathematics

Pl. Weyssenhoffa 11

85-072 Bydgoszcz

POLAND

E-mail address: kayalnabin82@gmail.com 